\title{
Sub-classification of non-inflammatory and inflammatory surgical aortic aneurysms and the association of histological characteristics with potential risk factors
}

\author{
DOINA BUTCOVAN ${ }^{1,2}$, VERONICA MOCANU $^{3}$, RALUCA ECATERINA HALIGA ${ }^{3,4}$, \\ BEATRICE GABRIELA IOAN ${ }^{5,6}$, MIHAI DANCIU ${ }^{2}$ and GRIGORE TINICA ${ }^{1,7}$
}

\begin{abstract}
${ }^{1}$ Department of Cardiovascular Surgery, "Prof George Georgescu" Institute of Cardiovascular Diseases, Iasi 700503; Departments of ${ }^{2}$ Morpho-Functional Sciences-Pathology, ${ }^{3}$ Morpho-Functional Sciences-Pathophysiology, ${ }^{4}$ Internal Medicine-Toxicology and ${ }^{5}$ Internal Medicine-Legal Medicine, 'Grigore T. Popa' University of Medicine and Pharmacy, Iasi 700115; ${ }^{6}$ Institute of Legal Medicine, Iasi 700455; ${ }^{7}$ Department of Surgery-Cardiac Surgery, "Grigore T. Popa" University of Medicine and Pharmacy, Iasi 700115, Romania
\end{abstract}

Received January 28, 2019; Accepted July 12, 2019

DOI: $10.3892 /$ etm.2019.7903

\begin{abstract}
The present study aimed to analyze the histological characteristics of surgical thoracic aortic aneurysm (TAA) and abdominal aortic aneurysm (AAA) specimens on the basis of the most recent consensus documents on non-inflammatory and inflammatory lesions. The current study also aimed to establish an association with various risk factors. Aortic wall specimens were collected from 52 patients ( 38 men and 14 women; age, 19-80 years) undergoing surgery for aortic dilatation at The Cardiovascular Disease Institute (Iasi, Romania). For histological evaluation, the aortic specimens (39 TAAs and 13 AAAs) were stained with hematoxylin-eosin, Van Giessen, alcian blue and Movat pentachrome. The specimens were evaluated and graded according to the severity of histopathological conditions: Fragmentation of elastic fibers, medial mucoid accumulation, smooth muscle cell loss and medial fibrosis. The severity of atherosclerotic lesions in surgically resected segments of the aorta were graded as follows: i) mild $=1$; ii) moderate $=2$; and iii) severe $=3$. The risk factors associated with TAA were the male sex (80\%), smoking (56\%), hypertension (33\%) and bicuspid aortic valve (13\%). Advanced age (70 years), male sex (69\%) and smoking (54\%) were determined to be the risk factors of AAA. The histopathological abnormalities included medial degeneration (MD) (82\%),
\end{abstract}

Correspondence to: Professor Veronica Mocanu, Department of Morpho-Functional Sciences-Pathophysiology, 'Grigore T. Popa' University of Medicine and Pharmacy, 16 Universitatii Street, Iasi 700115, Romania

E-mail: veronica.mocanu@umfiasi.ro

Key words: thoracic aortic aneurysm, abdominal aortic aneurysm, granulomatous or giant cell aortitis, non-infectious and infectious aortitis, Takayasu arteritis, consensus document, non-inflammatory, degenerative atherosclerosis (ATS) (42\%) and aortitis (10\%). MD was the leading histopathological diagnosis in TAA and the severity of lesions were graded as follows: Mild (8\% of cases), moderate ( $44 \%$ of cases) and severe (31\% of cases). Severe atherosclerotic lesions were identified in AAA (100\% of cases). In the present study, medial degenerative aortic lesions ( 1 , mild; 2 , moderate; and 3 , severe) significantly correlated with advanced age $(>65$ years; $\mathrm{r}=-0.39 ; \mathrm{P}<0.01)$ and male sex $(\mathrm{r}=0.27 ; \mathrm{P}<0.05)$. Significant correlations were also identified between atherosclerotic aortic lesions (1, mild; 2, moderate; and 3, severe) and advanced age ( $>65$ years) $(\mathrm{r}=-0.40, \mathrm{P}<0.01)$ or smoking $(r=-0.29 ; \mathrm{P}<0.05)$. Advanced age, male sex and smoking were determined to be the main risk factors for the development of degenerative aortic aneurysms.

\section{Introduction}

Diagnostics in aortic pathology require more attention $(1,2)$ and little is known about the histological patterns of aortic pathology with regard to medial degeneration (MD), atherosclerosis (ATS) and aortitis in relation to potential risk factors as well as to their distribution in different aortic segments. While MD is reported to be the leading histological finding in cases of aneurysm, the roles of ATS and inflammatory processes seem to be underestimated $(3,4)$. A clinical manifestation of all these aortic lesions is an aortic aneurysm. As a permanent dilatation of the aortic wall, an aortic aneurysm may affect all aortic segments, including the thoracic aorta and abdominal aorta (5). These aneurysms are classified according with involved aortic segments, such as aortic root, ascending aorta, arch, or descending aorta for thoracic aneurysms and suprarenal, juxtarenal and infrarenal aorta for abdominal aneurysms (1). The etiology, natural history, histology and treatment differ for each of these aneurysms segments, because the aorta is subject to a variety of diseases, degenerative, inflammatory, infectious and idiopathic (3).

The aim of the present study was to assess aortic aneurysms, including MD, ATS and inflammatory processes, according 
to the newest consensus documents on non-inflammatory and inflammatory aortic pathology (6-8) in association with potential risk factors.

\section{Materials and methods}

Patient characteristics. The intraoperative specimens of the entire aorta were obtained from 52 patients recruited between March 2015 and September 2016. The specimens included 39 thoracic aortic aneurysm (TAA) and 13 abdominal aortic aneurysm (AAA) samples from 38 men (73\%) and 14 women (27\%) with ages ranging between 19 and 80 years (average, 61.7 years). The specimens were recovered during open aortic surgery. The patients with TAA and severe aortic valve incompetence underwent ascending aortic replacement. Surgically, the sinotubular junction was remodelated using Dacron grafts and the aortic valve plane was relocated. The basic goal of surgical repair of AAA was the exclusion of the aortic aneurysm from the systemic circulation with preservation of blood flow to the pelvis and legs via an implanted new bifurcated graft (Hemashield Platinum).

The individual clinical data [age, sex, hypertension (HTA), smoking, history of the aortic disease and previous inflammatory processes] were obtained from medical records. The present study was conducted according to the Helsinki Declaration and approved by the Institutional Review Committee at The 'Prof. Dr. George Georgescu', Institute of Cardiovascular Diseases. Written informed consent was signed by all patients.

Histological analysis. For histological studies, AAT and AAA were fixed with $10 \%$ neutral buffered formalin solution (at room temperature, $24 \mathrm{~h}$ ) and embedded in paraffin. Cross-sections of aortic tissue $(4 \mu \mathrm{m})$ were stained with hematoxylin and eosin for tissue morphology. Elastic Van Gieson's staining (at room temperature, $55 \mathrm{~min}$ ) was used to analyze elastic fibers. The histological assessment was performed by an experienced pathologist using an optical microscope (CX41; Olympus Corporation; magnifications, $\mathrm{x} 40, \mathrm{x} 100, \mathrm{x} 200$ and $\mathrm{x} 400)$. The measurements were visualized using color image analysis software (QuickPHOTO MICRO 3.0; PROMICRA, s.r.o.).

To optimize the histological diagnosis, standardized nomenclature was applied, according to consensus documentation referring to unified nomenclature for a variety of non-inflammatory degenerative aortic lesions (8) for the histological analysis of the aorta specimens. ATS lesions were present in both TAA and AAA cases, presenting various degrees of severity with consequent aortic wall changes. The severity of the atherosclerotic lesions in surgically resected segments of the aorta was graded according to Stone et al (7): i) 1 , mild-1; ii) 2 , moderate; and iii) 3 , severe.

Statistical analysis. Data were analyzed using IBM SPSS Statistics 21 Software and results were expressed as mean \pm standard deviation and percentages. The correlations between non-inflammatory degenerative aortic lesions and potential atherosclerotic risk factors (age $>65$ years, male sex, smoking, arterial hypertension, and bicuspid aortic valve) were evaluated by Kendall's tau-b coefficient correlation with $\mathrm{P}<0.05$ being significant.

\section{Results}

An aneurysm is defined as a dilatation of $\geq 50 \%$ above the normal diameter of an artery. Of the total number of patients with aortic aneurysms who underwent aortic dilation, thoracic aorta was the most commonly involved segment $(n=39)$, while abdominal aorta was only identified in one-third of cases $(n=13)$; the two aortic segments demonstrated different etiologies. Aortic aneurysms have a multifactorial etiology. The multiple risk factors included advanced age, male, smoking, HTA, ATS and bicuspid aortic valve (BAV) (Table I).

The average patient age for TAA was 55.6 years in males and 50.6 years in females and the average patient age for AAA was 69.7 years in males and 71.2 years in females. From the entire aortic aneurysm group $(n=52)$, males were predominant in both the TAA group (79.5\%; 31 of 39 patients) and the AAA group $(69.2 \%$; 9 of 13 patients).

Of the risk factors, smoking was a more common finding in TAA (56.4\%; $n=22)$ than in AAA (46.2\%; $n=6)$. Similarly, HTA registered higher values in TAA $(33.3 \%$; $n=13)$ than in AAA $(23.1 \%$; $n=3)$. BAV was found only in five of the TAA cases $(12.8 \%)$ in association with aortic regurgitation. No genetic disorder history was identified in the study group patients.

Histopathological examination revealed only MD lesions (64.1\%, 25 of 39 patients), ATS lesions (23.1\%, 9 of 39 patients), mixed lesions, MD and ATS (17.9\%, 7 of 39 patients) in patients with TAA ( $\mathrm{n}=39)$, and microscopically proven aortitis $(12.8 \%$, 5 of 39 patients) in TAA, while in patients with AAA $(n=13)$ only ATS lesions (100\%) were present (Table I).

MD lesions in aortic aneurysms. A total of 32 (82.1\%) cases of MD (only lesions or together with ATS lesions) were identified in the patients with TAA. Of all these lesions, $3(12 \%)$ were with mild medial degeneration (mMD), 17 (43.6\%) with moderate medial degeneration (MMD) and 12 (48\%) with severe medial degeneration (SMD) (Fig. 1).

In the patients with TAA with mMD aortic lesions (grade 1), the aortic medial wall showed a multifocal decrease in the number of smooth muscle cells (SMCs) with minimal collagen deposition, a decrease in number of the elastic fibers (EF) and multifocal, mild EF fragmentation associated with focal, mild intralamellar mucoid accumulation (Fig. 1A).

In the patients with TAA with MMD aortic lesions (grade 2), the aortic wall had a decreased number of SMCs and multifocal mild substitutive fibrosis, a reduction in the number of EFs and multifocal moderate EF fragmentation with formation of multifocal, moderate intralamellar and focal, translamellar mucoid accumulation (Fig. 1B).

In the patients with TAA with SMD aortic lesions (grade 3) the medial aortic wall showed extensive, band-like SMC loss, multifocal moderate EF fragmentation and multifocal severe translamellar mucoid accumulation (Fig. 1C).

In our study, the medial degenerative aortic lesions (mild $=1$, moderate $=2$, and severe $=3$ ) significantly correlated with advanced age $(>65$ years $)(\mathrm{r}=-0.39, \mathrm{P}<0.01)$ and male sex $(\mathrm{r}=0.27, \mathrm{P}<0.05$; Table II).

Atherosclerotic lesions in aortic aneurysms. The atherosclerotic aneurysms were found in 22 patients, 13 (9 males, 
Table I. Comparative analysis of risk factors and histological characteristics in patients with surgical aortic aneurysms.

\begin{tabular}{lcccccc}
\hline Histological lesions & Patients n $(\%)$ & Age (years) & Male n $(\%)$ & HTA n (\%) & Smoking n (\%) & BAV n (\%) \\
\hline TAA & $39(100)$ & $54.6 \pm 13.7$ & $31(79.5)$ & $13(33.3)$ & $22(56.4)$ & $5(12.8)$ \\
Medial degenerative & $32(82.1)$ & $53.7 \pm 14.1$ & $28(87.5)$ & $12(37.5)$ & $19(59.4)$ & $5(15.6)$ \\
Mild & $3(7.7)$ & $48.7 \pm 23.0$ & $3(100)$ & $1(33.3)$ & $2(66.7)$ & $0(0)$ \\
Moderate & $17(43.6)$ & $52.9 \pm 16.1$ & $14(82.4)$ & $5(29.4)$ & $8(47.1)$ & $3(17.6)$ \\
Severe & $12(30.8)$ & $56.1 \pm 8.2$ & $11(91.7)$ & $6(50.0)$ & $9(52.9)$ & $2(16.7)$ \\
Atherosclerotic & $9(23.1)$ & $57.2 \pm 12.1$ & $6(66.6)$ & $1(33.3)$ & $1(16.7)$ & $0(0)$ \\
Mild & $6(15.4)$ & $57.0 \pm 14.1$ & $4(66.7)$ & $0(0)$ & $1(16.7)$ & $0(0)$ \\
Moderate & $3(7.7)$ & $57.8 \pm 8.7$ & $2(33.3)$ & $1(33.3)$ & $0(0)$ & $0(0)$ \\
Inflammatory & $5(12.8)$ & $54.4 \pm 12.6$ & $3(60)$ & $5(100)$ & $4(80)$ & $0(0)$ \\
TA & $1(2.6)$ & 36 & $0(0)$ & $1(100)$ & $1(100)$ & $0(0)$ \\
Syphilitic aortitis & $1(2.6)$ & 56 & $1(100)$ & $1(100)$ & $1(100)$ & $0(0)$ \\
GCA & $3(7.7)$ & $60.0 \pm 10.0$ & $2(66.7)$ & $3(100)$ & $2(66.7)$ & $0(0)$ \\
AAA & $13(100)$ & $70.2 \pm 8.0$ & $9(69.2)$ & $3(23.1)$ & $6(53.8)$ & $0(0)$ \\
Atherosclerotic lesions & $13(100)$ & $70.2 \pm 8.0$ & $9(69.2)$ & $3(23.1)$ & $6(53.8)$ & $0(0)$ \\
Severe & $13(100)$ & $70.2 \pm 8.0$ & $9(69.2)$ & $3(23.1)$ & $6(53.8)$ & $0(0)$ \\
\hline
\end{tabular}

TAA, thoracic aortic aneurysm; AAA, abdominal aortic aneurysm; HTA, hypertension; BAV, bicuspid aortic valve. GCA, giant cell aortitis; TA, Takayasu aortitis.
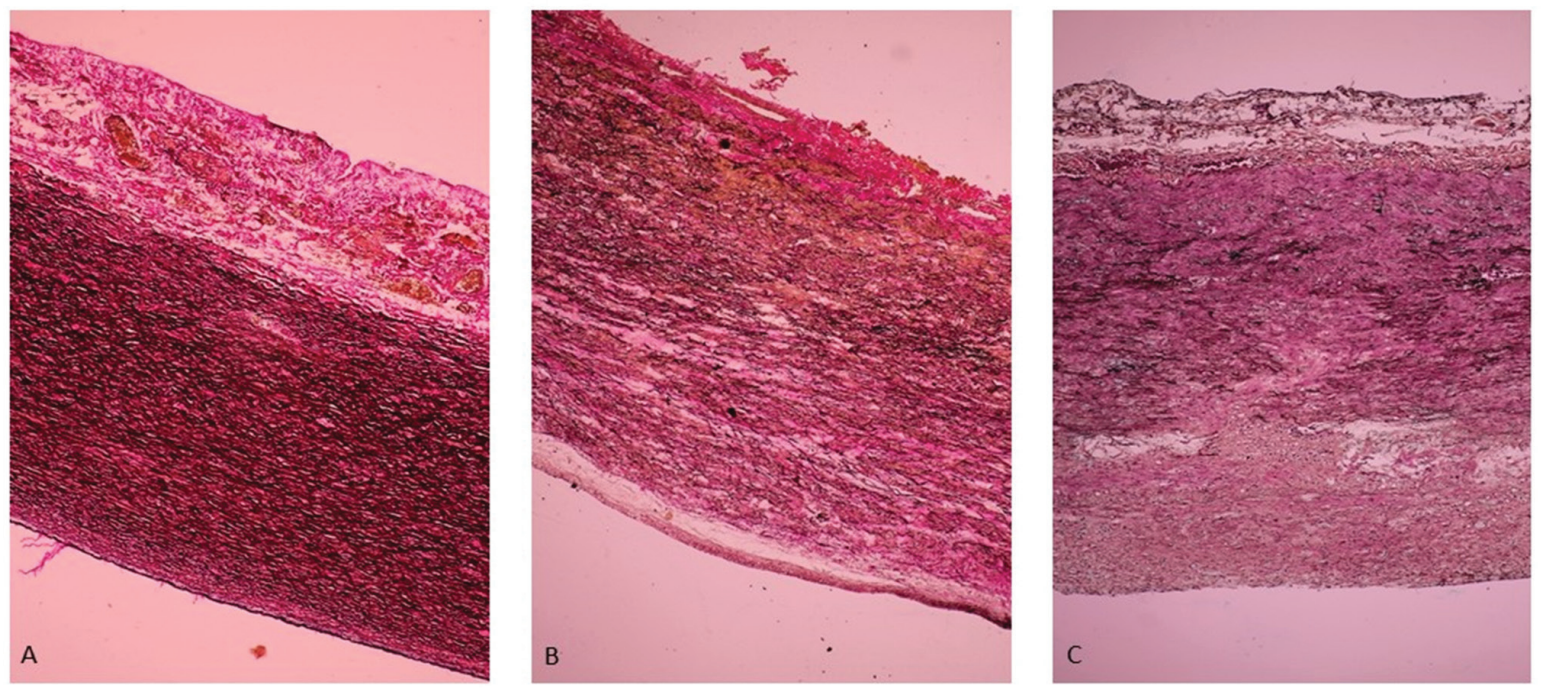

Figure 1. Aortic MD. Tissues of (A) grade 1, (B) grade 2 and (C) grade 3, were determined via Elastic Van Gieson's staining (magnification x100). MD, medial degeneration.

4 females) were abdominal and 9 (6 males, 3 females) were thoracic aneurysms. In the AAAs, all atherosclerotic lesions were severe (grade 3), with fibrotic changes of the entire wall, with or without associated complications. In the TAAs, most cases had MMD or SMD associated with intimal atherosclerotic lesions of different types (grades 1-2), with the formation of mixed, degenerative-atherosclerotic aneurysms (7 cases). A total of 2 patients with TAA with pure ATS lesions (mild) were identified. In most AAA cases, adventitial inflammation was also observed. The present results revealed early atherosclerotic lesions in the thoracic aorta (Fig. 2A) and advanced atherosclerotic lesions in the abdominal aorta (Fig. 2B).
Kendall's tau-b correlation shows that the atherosclerotic aortic lesions (1, mild; 2 , moderate; and 3 , severe) significantly correlated with advanced age $(>65$ years $)(r=-0.40, P<0.01)$ and smoking ( $\mathrm{r}=-0.29, \mathrm{P}<0.05$; Table II).

Inflammation in aortic aneurysms. In total, 5 patients with TAA had aortitis, determined by histological examination, including three giant cell aortitis (GCA) cases, one Takayasu aortitis (TA) case and one patient with syphilitic aortitis. The aortitis group included 3 men and 2 women. The GCA cases consisted of 2 males and 1 female with an average age of 62.5 years (ranging between 52 and 70 years); the patient with 
Table II. Kendall's Tau-b correlation coefficient of degenerative, atherosclerotic and inflammatory lesions with potential risk factors in aortic aneurysm $(\mathrm{n}=52)$.

\begin{tabular}{|c|c|c|c|c|}
\hline \multirow[b]{2}{*}{ Co-variables } & \multicolumn{2}{|c|}{$\begin{array}{l}\text { Medial degenerative } \\
\text { (mild, moderate, severe) }\end{array}$} & \multicolumn{2}{|c|}{$\begin{array}{c}\text { Atherosclerotic } \\
\text { (mild, moderate, severe) }\end{array}$} \\
\hline & r-value & P-value & $\mathrm{r}$-value & P-value \\
\hline Sex (Male) & 0.27 & $0.03^{\mathrm{a}}$ & -0.08 & 0.53 \\
\hline Age (>65 years) & -0.39 & $<0.01^{\mathrm{a}}$ & 0.40 & $0.02^{\mathrm{a}}$ \\
\hline Arterial hypertension & 0.22 & 0.10 & -0.18 & 0.17 \\
\hline Smoking & 0.13 & 0.34 & 0.29 & $0.03^{\mathrm{a}}$ \\
\hline
\end{tabular}

${ }^{\mathrm{a}} \mathrm{P}<0.05$. r, Kendall's Tau-b correlation coefficient.
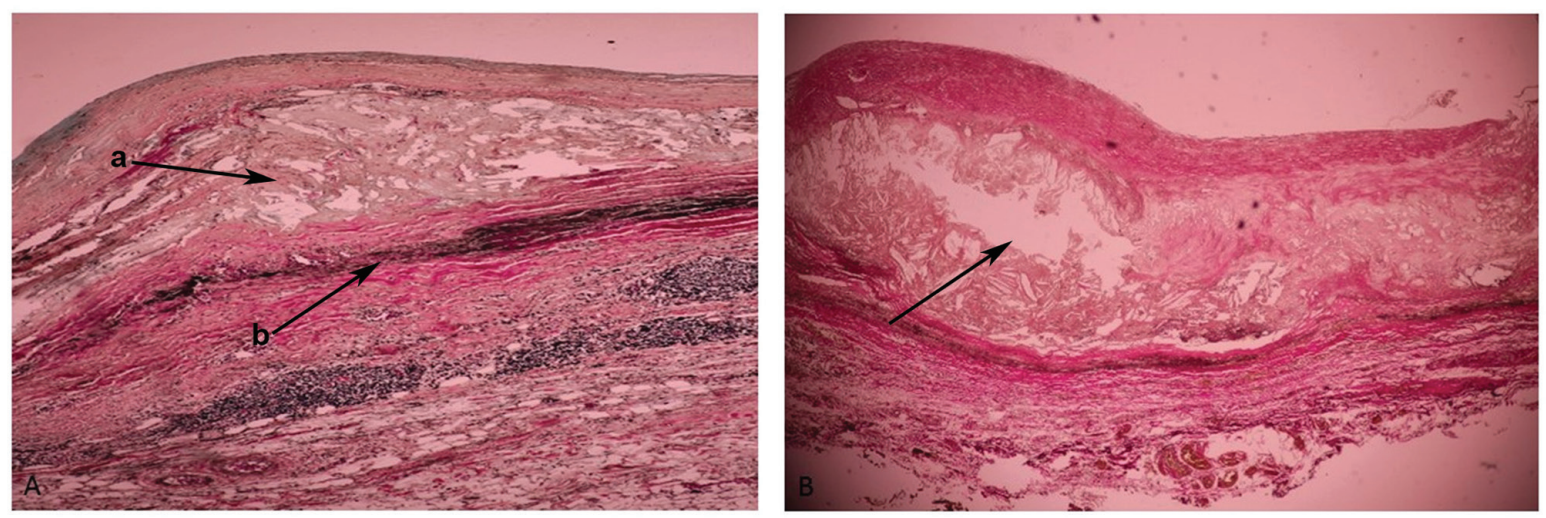

Figure 2. Aortic atherosclerotic aneurysms. Images of (A) mixed thoracic aortic and (B) atherosclerotic aortic aneurysms (black arrow, atherosclerotic plaque). Sections were examined via Elastic Van Gieson's staining (magnification, x100). a, atherosclerotic plaque; b, medial degenerative lesion.
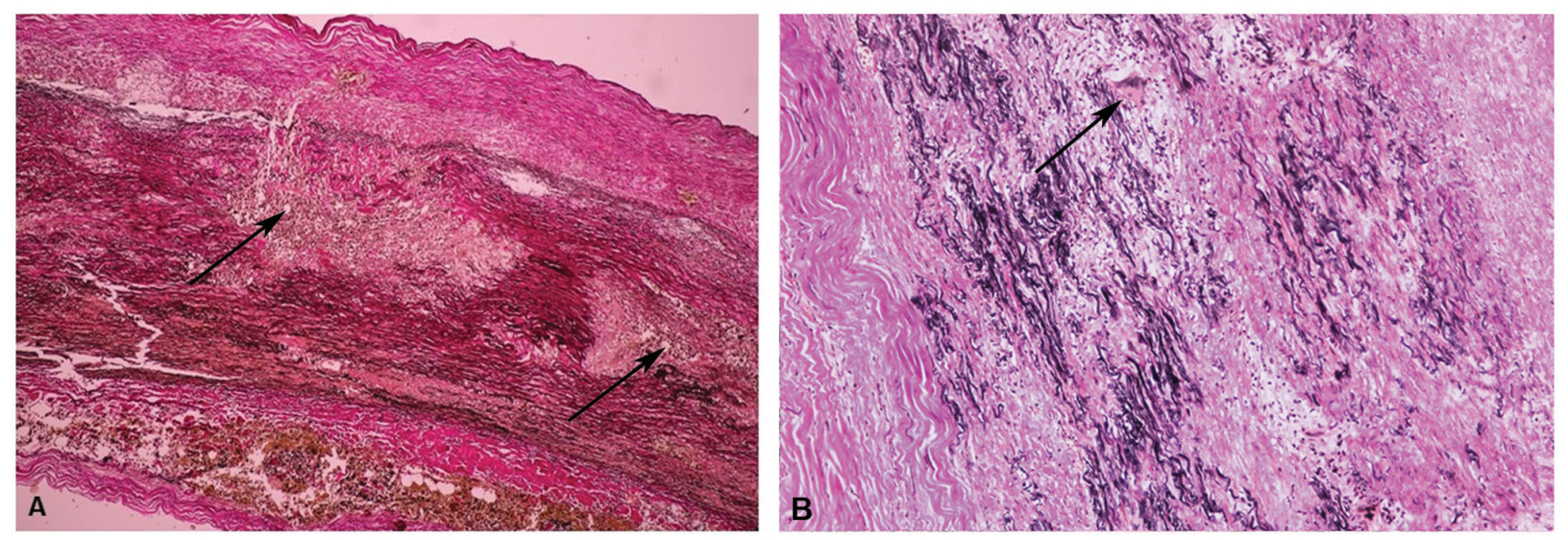

Figure 3. Non-infectious aortitis. (A) Giant cell aortitis with micronodular granulomas at the inner half of the media (arrows) and less adventitial fibrosis (determined via Elastic Van Gieson's staining; magnification, x100). (B) Takayasu aortitis with medial giant cell granuloma (arrow) and more adventitial fibrosis (determined via Elastic Van Gieson's staining; magnification, x400).

TA was a 36-year-old female and the patient with syphilitic aortitis was a 56-year-old male.

In all five TAA aortitis cases, the mean pre-operative erythrocyte sedimentation rate was $31.5 \mathrm{~mm} / \mathrm{h}$, and for C-reactive protein was $21.6 \mathrm{mg} / \mathrm{d}$. Of the 5 patients, $1(20 \%)$ needed coronary artery bypass grafting, $3(60 \%)$ underwent aortic valve surgery ( 1 aortic valve repair and 2 aortic valve replacement), and all $5(100 \%)$ required aortic surgery (ascending aortic replacement).

In most aortitis cases (60\%) the diagnosis was made after the operation based on histopathologic examination, except TA where the examination suggested pre-operatory TA disease, and syphilitic aortitis where the patient had a history of syphilis. 


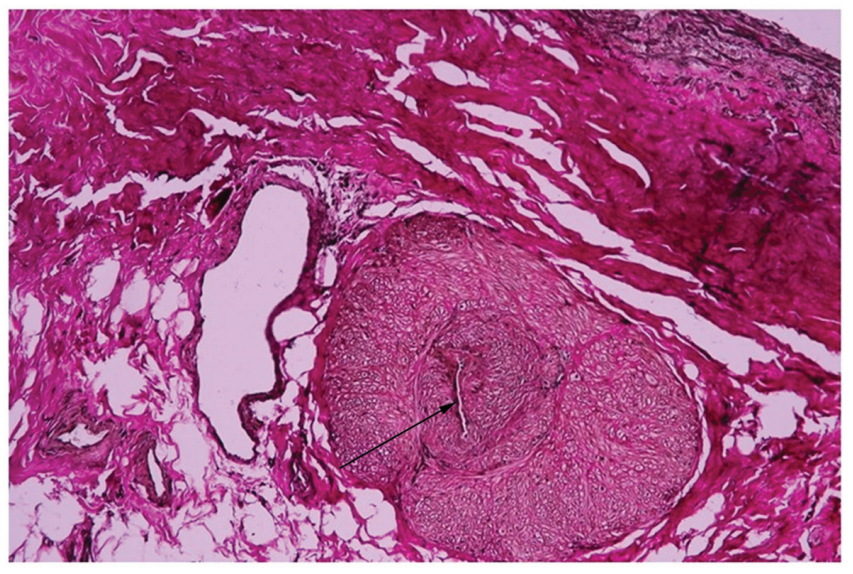

Figure 4. Endarteritis obliterans of the vasa vasorum and medial scar (determined via Elastic Van Gieson's staining; magnification, x100). The black arrow indicates adventiceal vasa vasorum with a very thick wall and slit-like stenotic lumen.

The common histological features in granulomatous aortitis were: i) Diffuse intimal hyperplasia, granulomatous inflammation in the media comprised of epithelioid macrophages and occasional giant cells related by 'laminar medial necrosis', areas of smooth muscle cell loss with collapsed elastic fibers, fibrotic scars and an adventitial accompanying lymphoplasmacytic infiltrate in GCA (Fig. 3A); and ii) diffuse intimal hyperplasia, compact granulomas and medial necrosis, scarring in the tunica media with disruption and disorganization of the remaining elastic fibers, and dense adventitial fibrosis in TA (Fig. 3B).

Among the 5 aortitis cases, one was diagnosed with syphilitic aortitis on the basis of the pathological features and patient history. A man in his $50 \mathrm{~s}$, who presented valvular regurgitation, aneurysmal disease and myocardial ischemia, also required coronary artery bypass grafting surgery. All these lesions were cardiovascular complications of syphilis involving the aorta (leading to the formation of aneurysms and aortic-valve incompetence) and coronary artery, causing coronary ostial stenosis (angina pectoris complaints). The histopathological examination revealed a chronic mesoaortitis, characterized by normal media replacement with fibrous scars and adventitial endarteritis of the vasa vasorum (Fig. 4).

\section{Discussion}

An aortic aneurysm is defined as a dilatation of an aortic segment with a diameter of at least one and one-half times the normal diameter (8). Aortic aneurysms can involve the ascending aorta or descending aorta. In the present study, the aortic aneurysms included $39(75 \%)$ thoracic and $13(25 \%)$ abdominal cases. Other previous epidemiology studies reported that AAA involves mainly older patients, while TAA involves mainly two populations: One of old age with no special history (9) and another of young age suffering from connective tissue disorders, including Marfan's syndrome (10). Marfan's syndrome or connective tissue disorders were not detected in the young patients with TAA in the present study $(11,12)$. Good familial history, histopathological examination and genetic screening are important factors that require consideration.
In agreement with Hiratzka et al (13), the present study identified that the most common risk factors for the development of aortic aneurysm were advanced age ( $>65$ years old), male, smoking, high blood pressure and family history. The potential risk factors identified to be associated with aortic aneurysm development were advanced age, HTA and BAV in TAA, and advanced age, male, smoking and ATS in AAA.

Previous studies reported that TAAs are most often associated with MD, which can be regarded as a structural alteration responsible for the development of aortic aneurysms in relation to other risk factors $(8,14)$. In the present study, the histopathological profile showed that MD was the most common histopathological substrate in TAA $(82.1 \%)$, granulomatous aortitis $(10.25 \%)$ was a rare cause of TAA and syphilitic aortitis $(2.5 \%)$ was a very rare encountered case, and ATS was a common histopathological substrate in AAA (100\%).

In patients with aortic aneurysms, histological analysis of the aortic resection specimens provides important diagnostic information on the MD lesions, describing a definite pattern of distribution of MD severity within the aortic wall. Moreover, aortitis was an unexpected diagnosis during the histological examination of surgically resected aortic thoracic aneurysms in patients without clinical signs or symptoms of systemic vasculitis. Aortic thoracic aneurysm is in most cases a fatal condition, especially in association with more severe degenerative changes in the aortic media.

GCA and TA, diagnosed in the present study, are considered the most common causes of non-infectious aortitis (8). Garcia-Martinez et al (15) observed that $\geq 20 \%$ of patients with GCA and $50 \%$ of patients with TA would develop changes consistent with aneurysmal aortitis, while in the present study, it was identified that only $7.6 \%$ of patients with GCA had TAA and $2.5 \%$ patients with TA had TAA, the remaining one TAA case having syphilitic aortitis.

Histopathological examination is not sufficient to distinguish between the two conditions, and, together with clinical data, it must be integrated within a diagnostic algorithm. In the final diagnosis, American College of Rheumatology (ACR) criteria were applied and at least three ACR criteria, including clinical, imagistic and histological data, were present to confirm the two granulomatous aortitis $(16,17)$.

In the present study, syphilitic aortitis was a rare cause of AA from all the TAA cases. In agreement with Vaideeswar (18), the complications in the form of aortic aneurysms and insufficiency in association with coronary ostial stenosis frequently coexist in the tertiary syphilis. For diagnosis, not only the pathological findings but also the clinical features and serology should be taken into consideration.

The present study also included 20 cases of atherosclerotic lesions. The clinical analysis of the 20 AAs revealed two types of aneurysmal diseases, 7 (35\%) mixed TAAs and $13(65 \%)$ ATS AAAs. The present results are in agreement with Singh et al (19), who noted that abdominal aneurysms account for the majority of aortic aneurysms. In the present study, the histopathological examination revealed intimal atheroma and parietal thrombosis in both AAA and aortic ATS, similar to the consensus statement on surgical pathology (20). The presence of ATS lesions in both groups may suggest the need for ATS medical therapy in all patients with TAA and AAA. Due to the highest degree of severity of the ATS AAA lesions, 
it was suggested that ATS plays an important role in AAA development.

In the present study, mixed TAA was associated with two lesions, MD and atherosclerotic lesions. However, additional marked elastin fragmentation and adventitial chronic inflammation were mainly restricted to AAA. Peshkova et al (21) identified that ATS changes in the aortic wall underlie AAA formation, where local inflammation contributes to aortic wall thickening. From the present results, it was hypothesized that abundant inflammatory infiltrate represents a common trigger for ATS and AAA development and extension. Additionally, the present results suggested that inflammation is a risk factor in AAA rupture, revealing the importance of anti-inflammatory medication in reducing AAA complications in patients.

The present study had a number of limitations, including a small group of patients with advanced aortic disease and no genetic studies. The small number of patients did not allow for more extensive statistical studies. The present study reflects morphoclinical particularities of aortic aneurysms in the geographical area. Further studies with larger biomarker panels are required to determine associations with other factors.

In conclusion, the present study provided insight for the development of aortic aneurysm evaluation for detecting and monitoring AAA and TAA in affected patients. The present study provides insight for the inclusion of histopathological examination in the final diagnosis, in consensus with the late knowledge and classification of aortic surgical pathology for diagnosis optimization. Prospective studies using large study groups will be necessary to further histologically evaluate the extent and severity of the disease and its progression.

\section{Acknowledgements}

Not applicable.

\section{Funding}

No funding was received.

\section{Availability of data and materials}

All data generated or analyzed during this study are included in this published article.

\section{Authors' contributions}

GT and DB conceived and designed the current study. DB, GT, $\mathrm{MD}$ and BGI performed the experiments. DB, MD, REH and VM collected and analyzed the data. DB, GT and VM wrote the manuscript. All authors read and approved the final version of the manuscript.

\section{Ethics approval and consent to participate}

The present study was performed in accordance with the ethical standards of the Declaration of Helsinki and was approved by the Research Ethics Committee of Professor George Georgescu Institute of Cardiovascular Diseases, Iasi, Romania (approval no. 974/17.02.2015). Informed consent was obtained from all individual participants included in the current study.

\section{Patient consent for publication}

Not applicable.

\section{Competing interests}

The authors declare that they have no competing interests.

\section{References}

1. Davies MJ: Aortic aneurysm formation: lessons from human studies and experimental models. Circulation 98: 193-195, 1998.

2. Elefteriades JA: Natural history of thoracic aortic aneurysms: Indications for surgery, and surgical versus nonsurgical risks. Ann Thorac Surg 74 (Suppl): S1877-S1880; discussion S1892-S1898, 2002.

3. Pomerance A, Yacoub MH and Gula G: The surgical pathology of thoracic aortic aneurysms. Histopathology 1: 257-276, 1977.

4. Stary HC, Chandler AB, Dinsmore RE, Fuster V, Glagov S, Insull W Jr, Rosenfeld ME, Schwartz CJ, Wagner WD and Wissler RW: A definition of advanced types of atherosclerotic lesions and a histological classification of atherosclerosis. A report from the Committee on Vascular Lesions of the Council on Arteriosclerosis, American Heart Association. Arterioscler Thromb Vasc Biol 15: 1512-1531, 1995.

5. Nesi G, Anichini C, Tozzini S, Boddi V, Calamai G and Gori F: Pathology of the thoracic aorta: A morphologic review of 338 surgical specimens over a 7-year period. Cardiovasc Pathol 18: 134-139, 2009.

6. Halushka MK, Angelini A, Bartoloni G, Basso C, Batoroeva L, Bruneval P, Buja LM, Butany J, d'Amati G, Fallon JT, et al: Consensus statement on surgical pathology of the aorta from the Society for Cardiovascular Pathology and the Association For European Cardiovascular Pathology: II. Noninflammatory degenerative diseases-nomenclature and diagnostic criteria. Cardiovasc Pathol 25: 247-257, 2016.

7. Stone JR, Bruneval P, Angelini A, Bartoloni G, Basso C, Batoroeva L, Buja LM, Butany J, d'Amati G, Fallon JT, et al: Consensus statement on surgical pathology of the aorta from the Society for Cardiovascular Pathology and the Association for European Cardiovascular Pathology: I. Inflammatory diseases. Cardiovasc Pathol 24: 267-278, 2015.

8. Leone O, Agozzino L, Angelini A, Bartoloni G, Basso C, Caruso G, D'Amati G, Pucci A, Thiene G and Gallo P: Criteria for histopathologic diagnosis of aortic disease consensus statement from the SIAPEC-IAP study group of 'cardiovascular pathology' in collaboration with the association for Italian cardiovascular pathology. Pathologica 104: 1-33, 2012.

9. Fritze O, Romero B, Schleicher M, Jacob MP, Oh DY, Starcher B Schenke-Layland K, Bujan J and Stock UA: Age-related changes in the elastic tissue of the human aorta. J Vasc Res 49: 77-86, 2012.

10. Iams HD: Diagnosis and management of Marfan syndrome. Curr Sports Med Rep 9: 93-98, 2010.

11. Topel I, Zorger N and Steinbauer M: Inflammatory diseases of the aorta: Part 1: Non-infectious aortitis. Gefasschirurgie 21 (Suppl 2): S80-S86, 2016.

12. Topel I, Zorger N and Steinbauer M: Inflammatory diseases of the aorta: Part 2: Infectious aortitis. Gefasschirurgie 21 (Suppl 2): S87-S93, 2016.

13. Hiratzka LF, Bakris GL, Beckman JA, Bersin RM, Carr VF, Casey DE Jr, Eagle KA, Hermann LK, Isselbacher EM, Kazerooni EA, et al: 2010 ACCF/AHA/AATS/ACR/ASA/ SCA/SCAI/SIR/STS/SVM guidelines for the diagnosis and management of patients with thoracic aortic disease: A report of the American College of Cardiology Foundation/American Heart Association Task Force on Practice Guidelines, American Association for Thoracic Surgery, American College of Radiology, American Stroke Association, Society of Cardiovascular Anesthesiologists, Society for Cardiovascular Angiography and Interventions, Society of Interventional Radiology, Society of Thoracic Surgeons, and Society for Vascular Medicine. Circulation 121: e266-e369, 2010.

14. Butcovan D: New frontiers in the histological diagnosis of degenerative aortopathies manifested as aortic aneurysms and dissections. Rev Med Chir Soc Med Nat 121: 89-94, 2017. 
15. Garcia-Martinez A, Hernandez-Rodriguez J, Arguis P, Paredes P, Segarra M, Lozano E, Nicolau C, Ramírez J, Lomeña F, Josa M, et al: Development of aortic aneurysm/dilatation during the followup of patients with giant cell arteritis: A cross-sectional screening of fifty-four prospectively followed patients. Arthritis Rheum 59: 422-430, 2008.

16. Park MC, Lee SW, Park YB, Chung NS and Lee SK: Clinical characteristics and outcomes of Takayasu's arteritis: Analysis of 108 patients using standardized criteria for diagnosis, activity assessment, and angiographic classification. Scand J Rheumatol 34: 284-292, 2005.

17. Ness T, Bley TA, Schmidt WA and Lamprecht P: The diagnosis and treatment of giant cell arteritis. Dtsch Arztebl Int 110: 376-386, 2013.

18. Vaideeswar P: Syphilitic aortitis: Rearing of the ugly head. Indian J Pathol Microbiol 53: 624-627, 2010.

19. Singh K, Bonaa KH, Jacobsen BK, Bjørk L and Solberg S: Prevalence of and risk factors for abdominal aortic aneurysms in a population-based study: The Troms $\varnothing$ study. Am J Epidemiol 154: 236-244, 2001
20. Stone JR, Basso C, Baandrup UT, Bruneval P, Butany J, Gallagher PJ, Halushka MK, Miller DV, Padera RF, Radio SJ, et al: Recommendations for processing cardiovascular surgical pathology specimens: A consensus statement from the standards and definitions Committee of the society for cardiovascular pathology and the association for European cardiovascular pathology. Cardiovasc Pathol 21: 2-16, 2011.

21. Peshkova IO, Schaefer G and Koltsova EK: Atherosclerosis and aortic aneurysm-is inflammation a common denominator? FEBS J 283: 1636-1652, 2016.

This work is licensed under a Creative Commons Attribution-NonCommercial-NoDerivatives 4.0 International (CC BY-NC-ND 4.0) License. 